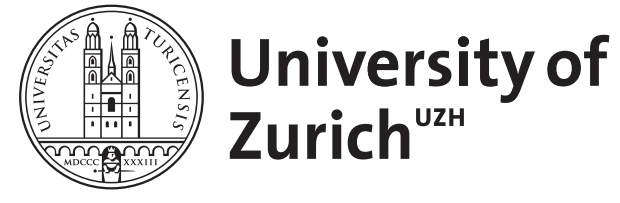

\title{
Aquaretic treatment in polycystic kidney disease
}

Wüthrich, Rudolf P ; Mei, Changlin

DOI: https://doi.org/10.1056/NEJMe1211857

Posted at the Zurich Open Repository and Archive, University of Zurich ZORA URL: https://doi.org/10.5167/uzh-70045

Journal Article

Published Version

Originally published at:

Wüthrich, Rudolf P; Mei, Changlin (2012). Aquaretic treatment in polycystic kidney disease. The New England Journal of Medicine, 367(25):2440-2442.

DOI: https://doi.org/10.1056/NEJMe1211857 
create an imbalance in the cytokine network, thereby driving or unmasking previously uncommon complications. The occurrence of pulmonary hypertension in one patient who received canakinumab and in two who received tocilizumab is notable, in light of a recent report of a significantly increased frequency of pulmonary hypertension in children with systemic JIA treated with a number of different therapies, including anti-interleukin- 1 and antiinterleukin-6.7 To address the challenge of evaluating long-term and infrequent adverse events that occur with these therapies, new approaches are needed, such as consolidated disease registries designed to evaluate safety signals in the context of the underlying disease and exposure to multiple agents.

It is interesting that the participants in these trials had marked similarities in the magnitude and timing of their responses, suggesting that interleukin-1 and interleukin- 6 may be in the same pathway in systemic JIA. In contrast, there is anecdotal evidence that some patients have a response to anti-interleukin- 1 but not to antiinterleukin-6, and vice versa, which is consistent with the complexity of proinflammatory cytokine networks. ${ }^{8}$ The partial or absent responses in a significant minority of patients in the trials of both canakinumab and tocilizumab suggest that genetic or environmental factors may result in different subsets of systemic JIA. Alternatively, there may be an early therapeutic window of opportunity during which higher response rates can be achieved,9 or inflammatory "escape" pathways may be activated because of the presence of these inhibitors. The search for the most proximal step that can be targeted in the inflammatory cascade will undoubtedly continue.

Despite important remaining questions about regulation of inflammation, the pathogenesis of systemic JIA, and appropriate interventions, there is no doubt that the agents tested in these trials signal a new era in the treatment of systemic JIA and will shed light on the mechanisms driving this enigmatic disorder. Continued investigation of systemic JIA is likely to inform our understanding of other multigenic autoinflammatory diseases - a growing category that now includes type 2 diabetes and inflammatory bowel disease - as well as our understanding of the regulation of inflammation.

Disclosure forms provided by the authors are available with the full text of this article at NEJM.org.

From the Department of Pediatrics, Stanford University School of Medicine, Stanford, CA.

1. Ruperto N, Brunner HI, Quartier P, et al. Two randomized trials of canakinumab in systemic juvenile idiopathic arthritis. N Engl J Med 2012;367:2396-406.

2. De Benedetti F, Brunner HI, Ruperto N, et al. Randomized trial of tocilizumab in systemic juvenile idiopathic arthritis. N Engl J Med 2012;367:2385-95.

3. Pascual V, Allantaz F, Arce E, Punaro M, Banchereau J. Role of interleukin-1 (IL-1) in the pathogenesis of systemic onset juvenile idiopathic arthritis and clinical response to IL-1 blockade. J Exp Med 2005;201:1479-86.

4. Verbsky JW, White AJ. Effective use of the recombinant interleukin 1 receptor antagonist anakinra in therapy resistant systemic onset juvenile rheumatoid arthritis. J Rheumatol 2004; 31:2071-5.

5. Yokota S, Miyamae T, Imagawa T, et al. Therapeutic efficacy of humanized recombinant anti-interleukin- 6 receptor antibody in children with systemic-onset juvenile idiopathic arthritis. Arthritis Rheum 2005;52:818-25.

6. Mellins ED, Macaubas C, Grom AA. Pathogenesis of systemic juvenile idiopathic arthritis: some answers, more questions. Nat Rev Rheumatol 2011;7:416-26.

7. Kimura YW, Haroldsom JE, Lee KL, et al. Pulmonary hypertension and other potentially fatal pulmonary complications in systemic juvenile idiopathic arthritis. Arthritis Care Res (Hoboken) 2012 November 8 (epub ahead of print).

8. Schmitz ML, Weber A, Roxlau T, Gaestel M, Kracht M. Signal integration, crosstalk mechanisms and networks in the function of inflammatory cytokines. Biochim Biophys Acta 2011;1813: 2165-75.

9. Nigrovic PA, Mannion M, Prince FH, et al. Anakinra as firstline disease-modifying therapy in systemic juvenile idiopathic arthritis: report of forty-six patients from an international multicenter series. Arthritis Rheum 2011;63:545-55.

DOI: $10.1056 /$ NEJMe1212640

Copyright (๑) 2012 Massachusetts Medical Society.

\title{
Aquaretic Treatment in Polycystic Kidney Disease
}

\author{
Rudolf P. Wüthrich, M.D., and Changlin Mei, M.D.
}

Autosomal dominant polycystic kidney disease (ADPKD), the most common hereditary kidney disease, progresses relentlessly to end-stage kidney disease before the age of 60 years in half the affected patients. One in 10 patients undergoing dialysis has ADPKD. The disease is characterized by prominent cystic enlargement of the kidneys, which is often associated with episodes of pain, 
hematuria, and cyst infection. An effective therapy to slow the progression of this disease does not exist, although several promising drug candidates are being tested. ${ }^{1}$

Vasopressin-mediated cyclic AMP (cAMP) generation is enhanced in polycystic kidneys, driving the proliferation of cyst epithelial cells and the secretion of cyst fluid. Tolvaptan, a selective vasopressin $\mathrm{V}_{2}$-receptor antagonist already in clinical use for the treatment of hyponatremia, decreased renal cAMP generation and target-gene expression in preclinical studies and was associated with delayed progression of ADPKD in a phase 2 clinical study. ${ }^{2}$

In the Tolvaptan Efficacy and Safety in Management of Autosomal Dominant Polycystic Kidney Disease and Its Outcomes (TEMPO) 3:4 trial, a large, phase 3 study now reported in the Journal, Torres et al. tested the efficacy and safety of tolvaptan in 1445 patients with ADPKD. ${ }^{3}$ At baseline, the mean age of the participating patients was 39 years. Kidney function was preserved, but nearly $80 \%$ of the patients had hypertension. Kidney size was increased by a factor of five, and half the patients had reported kidney pain. The patients were randomly assigned in a 2:1 ratio to receive two doses of tolvaptan or placebo daily.

The study met its primary and secondary end points - tolvaptan, when given at an average dose of $95 \mathrm{mg}$ per day over a 3-year period, slowed the usual increase in kidney volume by $50 \%$ and reduced the decline in the glomerular filtration rate (GFR). The effect of tolvaptan on kidney volume was most pronounced during the first year of the study, probably owing to the early reduction in the secretion of cyst fluid. ${ }^{4}$ In contrast, the effect on GFR became apparent only after the first year of treatment. A considerable number of patients discontinued tolvaptan owing to its aquaretic side effects (thirst, polydipsia, polyuria, and nocturia), and tolvaptan treatment was also associated with elevated liverenzyme levels, hypernatremia, an increased level of uric acid, and gout. However, as compared with patients who received placebo, significantly fewer patients who received tolvaptan had adverse events related to $A D P K D$, such as kidney and back pain, hematuria, and urinary tract infection.

The rate of increase in kidney volume was low in both study groups $(5.5 \%$ per year in the placebo group and $2.8 \%$ per year in the tolvaptan group), in contrast to growth rates of 6 to $12 \%$ per year in other studies. ${ }^{5,6}$ Because of the effect of tolvaptan on water excretion, the study design forced all patients to increase their fluid intake in order to avoid dehydration. Increased water intake to suppress vasopressin-mediated cAMP generation is recommended for patients with $\mathrm{ADPKD},{ }^{7}$ and the fact that participating patients followed this prescription could explain the reduced growth in kidney volume observed in this study. Nevertheless, tolvaptan, by driving fluid intake, slowed the expansion of cyst volume significantly more than placebo did.

Tolvaptan was associated with a slower decrease in kidney function and with less pain than placebo was, although the number of relevant events was low and contributed to less than $20 \%$ of the secondary outcome events. The study did not assess the effects of tolvaptan on kidney pain in a systematic fashion; thus, pain data need to be confirmed. Furthermore, it is unclear why tolvaptan had no effect on worsening trajectories of hypertension and albuminuria. Assuming that tolvaptan causes structural rather than functional improvements, one would have expected beneficial effects on urinary protein excretion and blood pressure.

Despite these criticisms, the TEMPO 3:4 study represents a major advancement in the quest for a cure for ADPKD. How might the information from this trial change the future care of patients with ADPKD? Would tolvaptan be a useful drug for the majority of such patients, or should it be prescribed only for those patients at greatest risk for progression to end-stage kidney disease? As Torres et al. comment, the use of this $\mathrm{V}_{2}$-receptor antagonist will depend on a balance between benefits and risks. The side effects of an aquaretic drug (e.g., thirst and polyuria) will affect the quality of life in a substantial number of patients - an issue that must be weighed against the possible benefits of delayed dialysis and transplantation, decreased kidney pain, and fewer urinary tract infections. Some patients with slowly progressive disease might opt for preemptive kidney transplantation (i.e., transplantation before the initiation of dialysis) rather than treatment with an aquaretic drug that has predictable, bothersome, long-term side effects. On 
the other hand, patients at risk for rapid progression might prefer treatment with an aquaretic agent and choose to cope with the side effects. The development of comprehensive criteria for aquaretic treatment and appropriate patient selection are needed, if tolvaptan is to be a successful therapy for patients with ADPKD.

Disclosure forms provided by the authors are available with the full text of this article at NEJM.org.

From the Division of Nephrology, University Hospital, Zurich, Switzerland (R.P.W.); and the Kidney Institute, Changzheng Hospital, Shanghai, China (C.M.).

This article was published on November 3, 2012, at NEJM.org.

1. Torres VE, Harris PC, Pirson Y. Autosomal dominant polycystic kidney disease. Lancet 2007;369:1287-301.
2. Higashihara $\mathrm{E}$, Torres $\mathrm{VE}$, Chapman $\mathrm{AB}$, et al. Tolvaptan in autosomal dominant polycystic kidney disease: three years' experience. Clin J Am Soc Nephrol 2011;6:2499-507.

3. Torres VE, Chapman AB, Devuyst O, et al. Tolvaptan in patients with autosomal dominant polycystic kidney disease. $\mathrm{N}$ Engl J Med 2012;367:2407-18.

4. Irazabal MV, Torres VE, Hogan MC, et al. Short-term effects of tolvaptan on renal function and volume in patients with autosomal dominant polycystic kidney disease. Kidney Int 2011;80: 295-301.

5. Serra AL, Poster D, Kistler AD, et al. Sirolimus and kidney growth in autosomal dominant polycystic kidney disease. $\mathrm{N}$ Engl J Med 2010;363:820-9.

6. Ruggenenti P, Remuzzi A, Ondei P, et al. Safety and efficacy of long-acting somatostatin treatment in autosomal-dominant polycystic kidney disease. Kidney Int 2005;68:206-16.

7. Torres VE, Bankir L, Grantham JJ. A case for water in the treatment of polycystic kidney disease. Clin J Am Soc Nephrol 2009;4:1140-50.

DOI: 10.1056/NEJMel211857

Copyright $\odot 2012$ Massachusetts Medical Society. 